Cahiers $d u$ MONDE RUSSE

\section{Cahiers du monde russe}

Russie - Empire russe - Union soviétique et États indépendants

45/3-4 | 2004

Varia

\title{
Recherches en multilinguisme et multiculturalité au siècle des Lumières
}

Séminaire international des jeunes dix-huitiémistes, château beuggen, allemagne, 12-17 septembre 2004

\section{VLADISLAV RÅEUCKIJ}

\section{CpenEdition}

\section{Journals}

Édition électronique

URL : https://journals.openedition.org/monderusse/8707

DOI : 10.4000/monderusse. 8707

ISSN : $1777-5388$

Éditeur

Éditions de l'EHESS

\section{Édition imprimée}

Date de publication : 1 juillet 2004

Pagination : 607-612

ISBN : 2-7132-2009-2

ISSN : $1252-6576$

Référence électronique

VLADISLAV RÅEUCKIJ, "Recherches en multilinguisme et multiculturalité au siècle des Lumières », Cahiers du monde russe [En ligne], 45/3-4 | 2004, mis en ligne le 01 janvier 2007, consulté le 04

septembre 2022. URL : http://journals.openedition.org/monderusse/8707 ; DOI : https://doi.org/ 10.4000 /monderusse. 8707 
chercher : repérer : avancer

Cet article est disponible en ligne à l'adresse :

http://www.cairn.info/article.php?ID REVUE=CMR\&ID NUMPUBLIE=CMR 453\&ID ARTICLE=CMR 4530607

Recherches en multilinguisme et multiculturalité au siècle des Lumières. Séminaire international des jeunes dix-huitiémistes, château beuggen, par VLADISLAV RÅEUCKIJ

\section{Editions de I'EHESS | Cahiers du monde russe}

2004/3-4 - Vol 45

ISSN 1252-6576 | ISBN 2713220092 | pages 607 à 612

Pour citer cet article :

—RÅEUCKIJ V., Recherches en multilinguisme et multiculturalité au siècle des Lumières. Séminaire international des jeunes dix-huitiémistes, château beuggen, allemagne, 12-17 septembre 2004, Cahiers du monde russe 2004/ 3-4, Vol 45 , p. $607-612$

Distribution électronique Cairn pour les Editions de l'EHESS.

(C) Editions de l'EHESS. Tous droits réservés pour tous pays.

La reproduction ou représentation de cet article, notamment par photocopie, n'est autorisée que dans les limites des conditions générales d'utilisation du site ou, le cas échéant, des conditions générales de la licence souscrite par votre établissement. Toute autre reproduction ou représentation, en tout ou partie, sous quelque forme et de quelque manière que ce soit, est interdite sauf accord préalable et écrit de l'éditeur, en dehors des cas prévus par la législation en vigueur en France. Il est précisé que son stockage dans une base de données est également interdit. 


\section{RECHERCHES EN MULTILINGUISME \\ ET MULTICULTURALITÉ \\ AU SIÈCLE DES LUMIÈRES}

\section{Séminaire international des jeunes dix-huitiémistes, château Beuggen, Allemagne, 12-17 septembre 2004}

Le séminaire qui a eu lieu courant septembre 2004 en Allemagne, au château de Beuggen, près de Bâle, est le quinzième du genre. Il s'est tenu sous la direction de Hans-Jürgen Lüsebrink (Universität Saarbrücken) et de Andres Kristol (Université de Neuchâtel) qui ont bénéficié de l'appui de la Société suisse d'étude du $\mathrm{XVIII}^{\mathrm{e}}$ siècle (SSEDS) et de son homologue allemand (DGEJ). Cette rencontre a réuni une quinzaine de jeunes docteurs et doctorants venant de presque dix pays européens. Les communications présentées à Beuggen ont permis de se faire une idée des recherches menées en multilinguisme et multiculturalité des Lumières actuellement. La problématique proposée portait sur l'étude des espaces multiculturels et multilinguistiques, les trajectoires biographiques de médiateurs culturels et linguistiques, les médias multiculturels et multilingues, le contact des langues et l'apprentissage des langues à travers l'histoire des institutions, des manuels, etc. L'image qui s'est dégagée des études présentées fut celle d'une Europe formée de sociétés en pleine mutation du fait de leur décloisonnement linguistique et culturel.

L'intensification des contacts entre différentes aires culturelles et linguistiques de 1'Europe, décrits en termes de transferts culturels ${ }^{1}$, autorise à parler d'une «République européenne des lettres ». Ce phénomène est fort complexe et inclut

1. Voir notamment Hans-Jürgen Lüsebrink, Rolf Reichardt, eds., en coll. avec Annette Keilhauer et René Nohr, Kulturtransfer im Epochenumbruch. Frankreich/Deutschland 1770-1815, Leipzig, Leipziger Universitätsverlag, 1996, 2 vols.; Barbara Schmidt-Haberkamp, Uwe Steiner et Brunhilde Wehinger, eds., Europäischer Kulturtransfer im 18. Jahrhundert : Literaturen in Europa - europäische Literatur ?, Berlin, Berliner Wissenschaftsverlag, 2003. 
des échanges non seulement bilatéraux, mais aussi tri- et multilatéraux d'une part ${ }^{2}$, et une dimension transatlantique d'autre part $^{3}$ (recherches du professeur Lüsebrink).

Une étude en multiculturalité et multilinguisme nécessite d'avoir une idée juste de la situation linguistique en Europe au XVIII siècle. Dans le cas de la France, outre la division du royaume en zones francophone et occitanophone, on peut observer une sorte de diglossie dans certaines couches de la population, par exemple dans la noblesse, bien obligée de maîtriser le patois ou le parler populaire pour se faire comprendre de ses serviteurs. Le français littéraire était l'apanage d'une petite minorité (quelques intellectuels, certains nobles, avocats des Parlements, etc.), en témoignent Les gasconismes corrigés par Lacour et Desgrouais (1803) ; 87 \% des erreurs relevées dans ce dictionnaire sont commises par des locuteurs appartenant aux couches supérieures de la société. Dans ce contrexte, l'apparition d'un espace francophone en France se présente comme un processus complexe de standardisation (recherches du professeur Andres Kristol).

Plusieurs études de cas présentées à ce séminaire appellent la comparaison. Tout en reconnaissant un contexte paneuropéen des Lumières, on a eu l'occasion de se faire une idée des variations locales, souvent très prononcées. Prenons comme exemple la traduction. Loin d'être simplement un moyen de diffuser des idées ou d'effectuer un transfert culturel, la traduction apparaît à cette époque comme un phénomène extrêmement complexe. Une ouvre écrite n'est pas considérée dans l'Europe des Lumières comme un monument, vision en somme assez récente, d'où des usages de la traduction très éloignés des nôtres. L'utilisation qu'en fait le baron d'Holbach (recherches d'Alain Sandrier, France ${ }^{4}$ ) est comparable à celle d'un traducteur hongrois anonyme du Spectator d'Adisson (dont le manuscrit a été découvert récemment par Gergely Labádi dans les archives de la bibliothèque de l'Académie de Kolozsvár en Roumanie, recherches de Peter Balasz, Hongrie) : l'un comme l'autre s'attachent à adapter le texte aux goûts de leurs publics, aux conditions et aux besoins du moment, soit en procédant à des modifications subtiles du texte source, soit en opérant un choix des chapitres à traduire. C'est ainsi que le Spectator dans la traduction hongroise se rapproche du genre à la mode en Hongrie,

2. Voir par exemple Michel Espagne, Katia Dimitrieva, eds., Philologiques IV. Transferts culturels triangulaires France-Allemagne-Russie, Paris, Éditions de la Maison des sciences de l'homme, 1996 ; et Hans-Jürgen Lüsebrink, « Trilateraler Kulturtransfer. Zur Rolle französischer Übersetzungen bei der Vermittlung von Lateinamerikawissen im Deutschland des 18. Jahrhunderts », in Günter Berger, Franziska Sick, eds., Französisch-deutscher Kulturtransfer im Ancien Régime, Tübingen, Stauffenberg Verlag, 2002, p. 81-97.

3. Voir notamment les travaux publiés sous la direction de H.-J. Lüsebrink, sous le titre « Transferts culturels entre l'Europe et l'Amérique du Nord aux XVIII et XIXe siècles. Circulation des savoirs, réappropriations formelles, réécritures », Tangence, 72, Été 2003 (Presses universitaires du Québec) ; Laurier Turgeon, Denys Delâge et Réal Ouellet, eds., Transferts culturels et métissages, Amérique/Europe, $X V I^{\mathrm{e}}-X X^{\mathrm{e}}$ siècles, Québec - Paris, Presses de l'Université Laval - L'Harmattan, 1996.

4. Auteur de l'ouvrage Le style philosophique du baron d'Holbach. Conditions et contraintes du prosélytisme athée en France dans la seconde moitié du XVIII siècle, Paris, H. Champion, 2004. 
les méditations pieuses, et s'éloigne de l'original d'autant plus que le traducteur se permet de « suivre le sens, parfois aux dépens de la lettre » et de traduire non pas à partir de l'original anglais mais à partir de la traduction française. Le même phénomène d'adaptation de l'œuvre par le traducteur est connu dans les espaces russe (voir les traductions des articles de l'Encyclopédie en russe où la censure se faisait tant au niveau du choix des articles qu'à celui du texte ${ }^{5}$ ) et allemand (voir par exemple les travaux de Bernhard Fabian) ${ }^{6}$.

Étroitement liée à cette question de la traduction est celle de la réception des littératures européennes dans divers pays d'Europe, que ce soit le roman anglais en territoires italiens (objet d'étude de Sandra Parmegiani, Italie/Canada) ou le roman français dans l'espace allemand (recherches de Nathalie Ferrand, France ${ }^{7}$ ). Ces recherches sont menées dans la mouvance des études de Maria Roza Zambon sur la réception du roman français en Italie, de celles d'Isabel Herrero, Lydia Vasquez et Dolorès Himenez sur le sort de la littérature française en Espagne ou encore de celles de Bernhard Fabian sur la réception de la littérature britannique en Allemagne. Les moyens d'étudier ces questions sont par définition très variés car évaluer l'importance d'un auteur ou d'un livre dans un espace culturel est une tâche ardue et suppose l'exploration des bibliothèques et des catalogues privés, le recensement des éditions, l'analyse des critiques littéraires, etc. Ainsi Nathalie Ferrand répertorie les traductions du roman français dans la Herzogin Anna Bibliothek de Weimar, en accordant une attention particulière à la provenance des livres, à leurs lecteurs, aux notes marginales. On constate la diversité géographique de cette réception en passant de la presse vénitienne, plutôt favorable aux auteurs britanniques, aux journaux et revues de Florence, de Rome ou de Milan qui font preuve de beaucoup plus de réserve, voire de rejet. La naissance de l'idéologie et de l'identité nationales italiennes se reflète aussi dans ces critiques littéraires.

Par ailleurs les auteurs français les plus traduits ne sont pas forcément les mêmes d'un pays à l'autre malgré le contexte paneuropéen (le cas hollandais est étudié par Edwin van Meerkerk, Pays-Bas) ce qui peut s'expliquer par la situation linguistique et culturelle spécifique du pays concerné. En effet, en Hollande par exemple, le public était en partie francophone et les éditions originales en français étaient largement accessibles. La donne n'est évidemment pas la même du côté de Saint-Pétersbourg et de Moscou où le français était beaucoup moins parlé et l'influence allemande était très considérable (voir notamment les travaux de Vladimir Somov et

5. Voir par exemple Sergej Karp, Francuzskie prosvetiteli i Rossija. Issledovanija i novye materialy po istorii russko-francuzskih kulturnyh svjazej vtoroj poloviny XVIII veka (Les philosophes français des Lumières et la Russie. Recherches et nouveaux matériaux pour l'histoire des relations culturelles russo-françaises de la seconde moitié du XVIII siècle), Moscou, RAN, 1998, p. 13-31.

6. Pour le problème de la censure dans la traduction, voir aussi les travaux réunis sous la direction de Denise Merkle dans le numéro intitulé «Censure et traduction dans le monde occidental » de la revue TTR : traduction, terminologie, rédaction, vol. XV, 2, 2002. Pour la question de la traduction comme outil de la médiation culturelle, voir aussi H.-J. Lüsebrink, «Trilateraler Kulturtransfer. ... », art. cit., p. 81-97.

7. Auteur de Livre et lecture dans les romans français du XVIII siècle, Paris, PUF, 2002. 
Nikolaj Kopanev). Dans l'empire habsbourgeois le commerce du livre forme un réseau de communication d'autant plus important qu'il s'agit d'une société morcelée du point de vue de la langue et de la culture. C'est un sujet relativement peu étudié, mais sa signification est bien comprise par les chercheurs dont le projet Der Buchmarkt der Habsburgermonarchie (1750-1850) a été présenté au séminaire par Johannes Frimmel (Autriche). Il s'agit d'un projet du Fonds autrichien pour la promotion de la recherche scientifique (FWF, directeur du projet : Peter R. Frank).

Le français était largement pratiqué aussi au royaume de Suède, s'inscrivant dans le contexte d'un fort multilinguisme et multiculturalisme (le suédois, l'allemand, le finlandais étaient parlés par différentes couches de la population en Suède). Les recherches de Charlotta Wolff (Finlande) montrent que la diffusion de la culture et de la langue françaises était pour la noblesse suédoise facilitée par le caractère transfrontalier, multilingue et cosmopolite qui lui était propre. Les moyens de la francisation des élites en Suède (apprentissage du français à la maison et dans les pensionnats éducatifs, voyages - y compris le Grand Tour - et le service à l'étranger, etc.) sont en grande partie les mêmes qu'en Russie. À ces moyens classiques, il faut ajouter les réseaux de sociabilité qui permettaient à un seigneur russe d'avoir une multitude de contacts avec les Français présents en Russie, dans des cadres très variés, créant ainsi un véritable bain culturel (voir nos propres recherches, Russie/France). Par ailleurs on peut observer que les précepteurs et les pensionnats français sont nombreux à Saint-Pétersbourg, mais l'édition des manuels de français, notamment des manuels pour les autochtones, y connaît un retard considérable par rapport à la Suède ${ }^{8}$. Notons au passage que si la maîtrise de l'allemand par les élites hongroises ouvrait la voie à l'influence directe des Lumières allemandes dans ce pays, la relative méconnaissance du français contribua à l'apparition de nombreuses traductions du français.

La question du développement linguistique des populations en contact occupe nombre de chercheurs, citons à ce propos les contributions d'Ulrich Müller (Allemagne) qui s'intéresse à la situation des populations sur la frontière germano-polonaise, ou celle de Gunilla Eschenbach (Allemagne) qui étudie le multilinguisme comme un moyen de satire et de critique dans les opéras allemands, plus particulièrement à l'opéra de Hambourg. En ce qui concerne ce dernier thème de recherche, la compétence linguistique du public de cet opéra était prise en compte par les auteurs des libretti qui mettaient en scène, à des fins satiriques, des personnages parlant le français, l'italien, le danois, etc. Manuella Böhm (Allemagne) s'intéresse, elle aussi, au problème des contacts linguistiques et culturels ; ses recherches portent sur l'acculturation et le changement de langue chez les réfugiés protestants à Berlin au XVIII ${ }^{\mathrm{e}}$ siècle. Dans le cas des huguenots de Berlin, l'étude des changements linguistiques peut être productive car beaucoup de documents officiels et privés rédigés par eux nous sont parvenus. On peut recourir à une fine analyse

8. Le cas de la Suède est étudié dans les livres de Elisabet Hammar, notamment : L'enseignement du français en Suède jusqu'en 1807 : méthodes et manuels, Stockholm, Akademilitteratur 1980. 
linguistique de ces témoignages pour avoir une meilleure idée de la diglossie qui se développait dans cette colonie huguenote, mais l'exercice reste néanmoins extrêmement délicat. On peut aussi constater une forte résistance à l'assimilation dans ce milieu, phénomène complexe qui ne s'explique pas que par le prestige attaché au français au siècle des Lumières. L'exemple de la communauté catholique française en Russie montre que l'assimilation dépend d'une multitude de critères : le niveau social des immigrés, les possibilités d'intégration, les différences confessionnelles, culturelles et linguistiques, la réussite ou l'échec du dialogue interculturel, etc.

Dans l'Europe des Lumières une place importante revient à la figure du médiateur culturel et linguistique. Il s'agit de personnes qui par leurs compétences facilitaient la circulation des idées et des objets entre différentes aires culturelles. Ces médiateurs sont évidemment nombreux à l'époque où des dizaines de milliers de personnes partaient en voyage ou s'expatriaient en Europe. Les œuvres scientifiques et littéraires servent aussi la même cause de médiation entre les cultures. Le prince Dimitrie Cantemir (1673-1723), père du diplomate et poète russe du même nom, longtemps exilé à Constantinople avant de trouver refuge en Russie, compte parmi les meilleurs spécialistes de l'histoire de l'Empire ottoman et de la culture des Turcs. Ce qui le place parmi les grands médiateurs culturels, c'est surtout la confrontation qu'il fait entre les points de vue des historiens occidentaux et orientaux, sans précédent à cette époque ; et ses œuvres offrent une étude de l'islam beaucoup plus poussée que ce qui se pratiquait alors en Europe. Sa mission de médiation fut sans doute facilitée par son élection, en 1714, à l'Académie de Berlin (recherches de Christina Birsan). Montesquieu peut aussi être compté parmi les médiateurs culturels des Lumières, et à différents titres ; comme voyageur, il se rendit en Angleterre, comme auteur, il se documenta beaucoup sur ce pays, notamment dans la presse britannique contemporaine, et évoqua les libertés britanniques dans ses œuvres. Il exerça aussi une influence certaine sur cette même presse qui multiplia les imitations de ses Lettres persanes (recherches d'Ursula Haskins, Grande-Bretagne).

Les contributions à ce séminaire seront publiées par les Éditions Champion/ Slatkine dans la série «Études du XVIII ${ }^{\mathrm{e}}$ siècle ».

rjeoutski@free.fr 